\title{
Endoscopic treatment of esophageal fistulas after esophagectomy with injection of an alpha-cyanoacrylate monomer: a phase II study $\square$
}

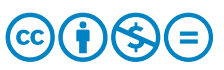

\author{
Authors \\ Toshiyasu Ojima, Masaki Nakamura, Mikihito Nakamori, Masahiro Katsuda, Keiji Hayata, Toshiaki Tsuji, \\ Shimpei Maruoka, Hiroki Yamaue
}

Institution

Second Department of Surgery, Wakayama Medical

University, Wakayama, Japan

submitted 14.12 .2017

accepted after revision $\quad 2.2 .2018$

Bibliography

DOI https://doi.org/10.1055/a-0581-9005 |

Endoscopy International Open 2018; 06: E1093-E1099

(c) Georg Thieme Verlag KG Stuttgart · New York

ISSN 2364-3722

Corresponding author

Hiroki Yamaue, MD, Second Department of Surgery, School of Medicine, Wakayama Medical University, 811-1,

Kimiidera, Wakayama 641-8510, Japan

Phone: 81-73-441-0613

Fax: 81-73-446-6566

yamaue-h@wakayama-med.ac.jp

\section{ABSTRACT}

Background and study aims Interventional endoscopic treatments including the application of glue are becoming more frequently used for the treatment of esophageal fistulas. However, there are no prospective studies of endoscopic treatment for esophageal fistulas. This prospective study aims to investigate the efficacy and safety of endoscopic injection of alpha-cyanoacrylate monomer for intractable esophageal fistulas.
Patients and methods This single-center prospective phase II trial included patients with more than 1 wk of conservative medical treatment for intractable esophageal fistulas after esophagectomy. In the image-guided therapy suite, a mixture of alpha-cyanoacrylate monomer and oily contrast agent in a ratio of 0.3 to $1.7 \mathrm{~mL}$ was endoscopically injected through the fistula.

Results Twenty-five patients who underwent esophagectomy at Wakayama Medical University Hospital were enrolled in this study. The primary disease was esophageal cancer in 16 patients (64\%) and gastric cancer in the remaining 9 patients (36\%). Complete closure of the esophageal fistula was performed in 22 patients after endoscopic injection of alpha-cyanoacrylate monomer. The overall success rate was $88 \%$. There was no fistula recurrence in any successful closure cases. Three patients with failed esophageal fistula closure had esophageal cancer with cervical esophageal fistulas and required reoperation of the fistulectomy under general anesthesia. No complications associated with this endoscopic treatment were detected.

Conclusions Endoscopic treatment with injection of alpha-cyanoacrylate monomer facilitated healing of postesophagectomy fistula in $88 \%$ of patients without complications. This suggests that the treatment is effective and safe for patients with esophageal fistulas.

University Hospital Medical Information Network UMIN000018486

TRIAL REGISTRATION: randomized clinical trial UMIN000018486 at http://www.umin.ac.jp

\section{Introduction}

latrogenic esophageal fistula after esophagectomy is a serious complication for esophageal and gastric cancers. It can lead to longer hospital stay and high postoperative morbidity and mortality rates. As esophageal fistula is refractory, management of it is often challenging.
Interventional endoscopic treatments including the application of glue and endoscopic clips and stent insertion are increasingly used for treatment of esophageal fistulas [1]. A main limitation of covered stent is the risk of migration, with several studies identifying rates more than $50 \%$ [2,3]. On the other hand, application of glue has become a promising method in clinical practice for avoiding reoperation after gastro- 
intestinal leakage $[1,4]$. The use of cyanoacrylate glue was first reported in 1983 as a treatment for tracheoesophageal fistula [5]. Since then, there have been many case reports of successful fistula closures by using glue [1, 6-10]. In many of these reports, n-butyl cyanoacrylate polymer or 2-octyl cyanoacrylate polymer was used via endoscopic injection [5-7]. We propose that alpha-cyanoacrylate ( $\alpha$-CA) monomer is ideal for glue embolization against intractable esophageal fistulas because $\alpha$-CA monomer glue spreads faster and thus creates a bond more rapidly than the other polymer glues $[1,10,11]$. We previously demonstrated 4 case series with esophageal fistulas that were successfully treated with endoscopic injection of $\alpha$-CA monomer [12]. Excluding our preliminary report, only 1 case report has so far examined the usefulness of $\alpha$-CA monomer for the treatment of esophageal fistulas [8].

This prospective phase II trial aims to test the hypothesis that endoscopic injection of $\alpha-C A$ monomer is effective and safe in patients with intractable esophageal fistulas. We planned this study to apply the endoscopic treatment in a clinical setting.

\section{Patients and methods}

\section{Eligibility criteria}

This study was conducted according to a protocol reviewed and approved by the Wakayama Medical University Hospital Ethical Committee on Human Research. All patients gave written informed consent before enrolling in the study. The study protocol was registered at the University Hospital Medical Information Network (UMIN000018486).

Participants were enrolled in the trial between January 2015 and March 2017 and regarded as eligible if they met the following criteria: (i) aged between 20 and 85 years; (ii) invalid cases with more than $1 \mathrm{wk}$ of conservative medical treatment for intractable esophageal fistulas; (iii) had no severe disturbance of liver or renal functions, as indicated by aspartate aminotransferase and alanine aminotransferase levels of $<300 \mathrm{lU} / \mathrm{L}$, and creatinine level of $<3.0 \mathrm{mg} / \mathrm{dL}$. Exclusion criteria were as follows: (i) patients with esophagotracheal fistulas; (ii) patients with pulmonary fistulas; (iii) patients with shock vital.

\section{Surgical procedure (video)}

Details of the endoscopic treatment methods performed at our institute have been previously described [12]. In brief, all patients were treated with endoscopic injection of $\alpha$-CA monomer glue. Endoscopic treatments were performed while patients were under sedation induced by intravenous diazepam. Patients were placed in the left decubitus position. We used a standard endoscope with a single accessory channel (GIF H260; Olympus, Tokyo, Japan). In the image-guided therapy suite, a mixture of $\alpha$-CA monomer (Aron Alpha A; Sankyo, Tokyo, Japan) and oily contrast agent (Lipiodol; Guerbet, Tokyo, Japan) in a ratio of 0.3 to $1.7 \mathrm{~mL}$ was endoscopically injected through the fistula using a dispersion tube and $2.5-\mathrm{mL}$ glue syringe ( $\triangleright \mathbf{F i g} . \mathbf{1}$ ). We used $50 \%$ glucose to push the solution from the syringe into the dispersion tube ( $\nabla$ Fig. $\mathbf{1}$ ). We repeated this procedure every 1 or 2 wk until the fistula was closed.

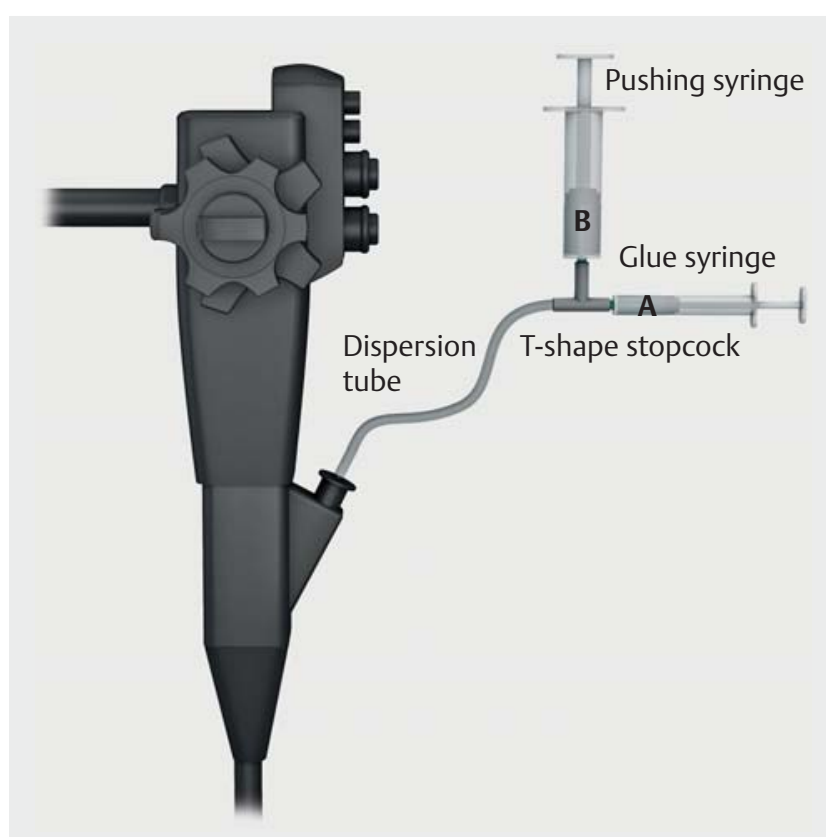

- Fig. 1 Schematic drawing of the endoscopic injection of cyanoacrylate glue. a Glue syringe: a mixture of cyanoacrylate and oily contrast agent (lipiodol) in a ratio of 0.3 to $1.7 \mathrm{~mL}$ in a $2.5-\mathrm{mL}$ syringe. b Pushing syringe: $5 \mathrm{~mL}$ of $50 \%$ glucose in a $10-\mathrm{mL}$ syringe.

\section{Study design}

We previously confirmed safety of this endoscopic treatment [12]. In this study, we adopted a phase II study design to evaluate the response rate. The primary endpoint of this study was to assess the objective response rate (fistulas closure rate). According to endoscopic findings $4 \mathrm{wk}$ after the endoscopic treatment, we considered the patients with reduction of the esophageal fistula orifice to be successful cases.

Secondary endpoints were to evaluate treatment-related toxicity and the late complications rate. Postoperative complications were analyzed according to the Clavien-Dindo classification [13]; complications higher than grade II were regarded as clinically significant. The planned sample size of the phase II study was 25 patients, a number that was required to confirm the null hypothesis that the $95 \%$ confidence interval of the expected overall response rate (85\%) would be less than $60 \%$ under the condition of $\alpha$-error of 0.05 and $\beta$-error of 0.2 .

\section{Results}

\section{Patients}

Twenty-five invalid patients with more than $1 \mathrm{wk}$ of conservative medical treatment for intractable esophageal fistulas were enrolled in this study between January 2015 and March 2017.

- Table 1 shows the clinical characteristics and surgical outcomes of the 25 patients (male: 22 , female: 3 ), with a median age of 69 years (range: $47-83$ years). The primary disease was esophageal cancer in 16 patients (64\%), and the cause of the esophageal fistula of these 16 patients was anastomotic leak- 

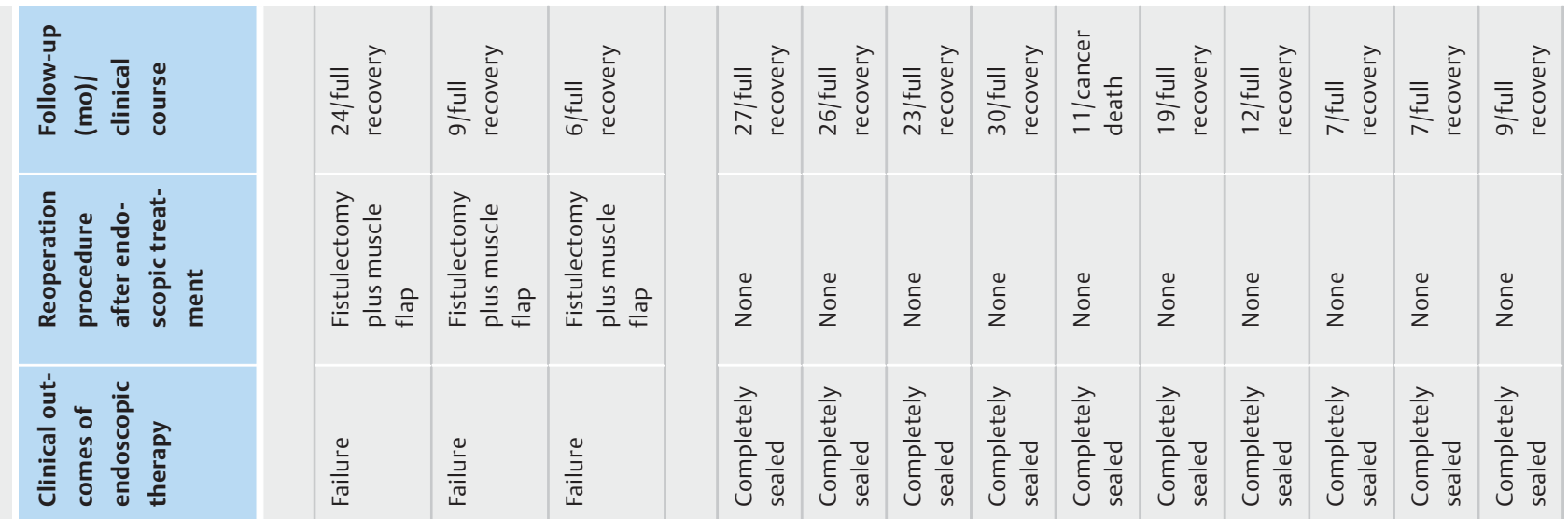

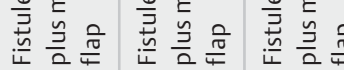

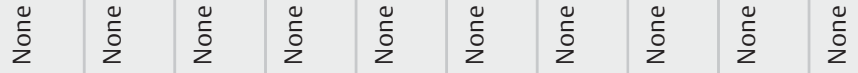

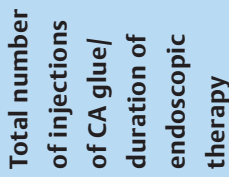

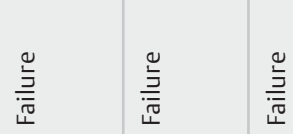

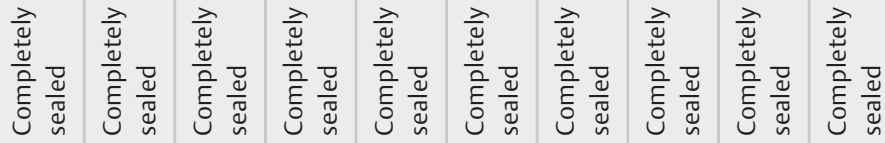

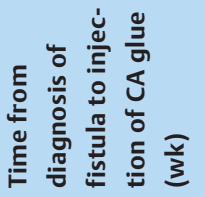

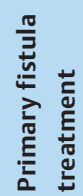

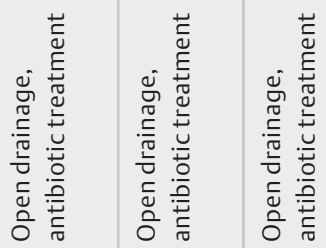

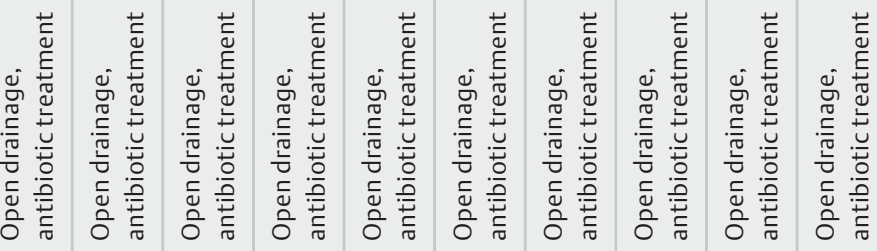

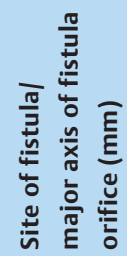

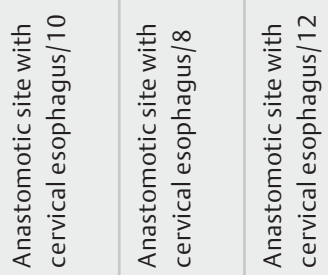

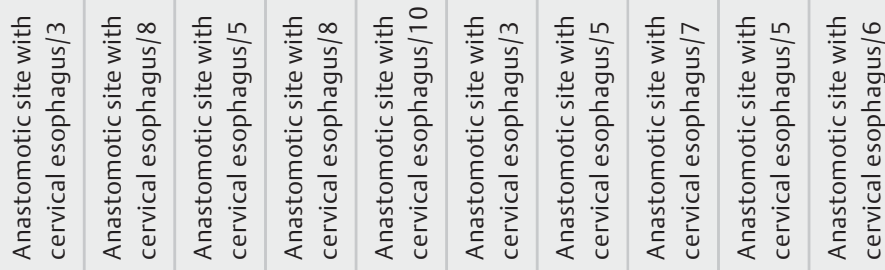

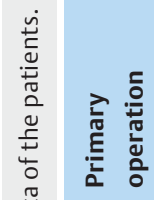

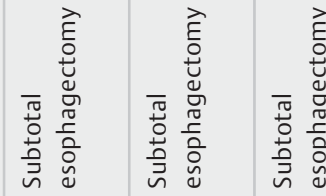

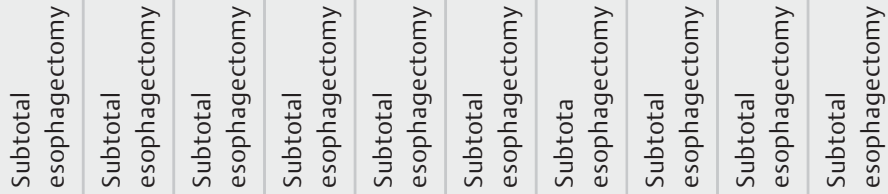

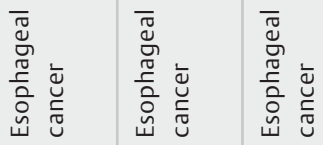

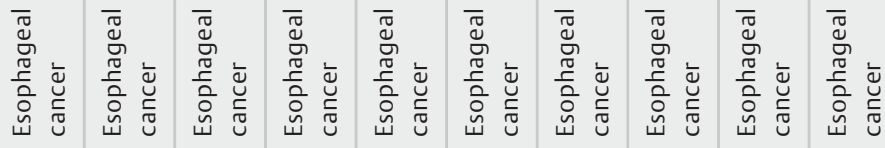
突 ๑ั

든

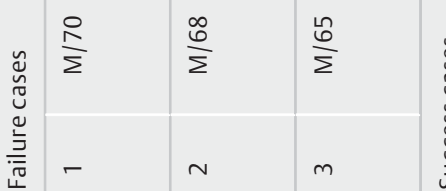

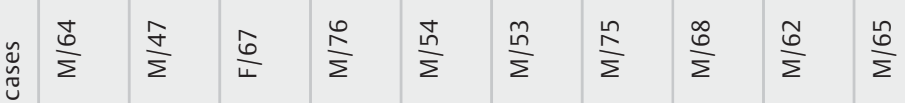
$\stackrel{20}{3}$ 


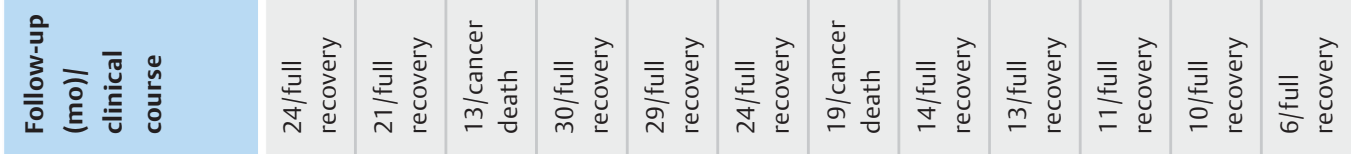

Min

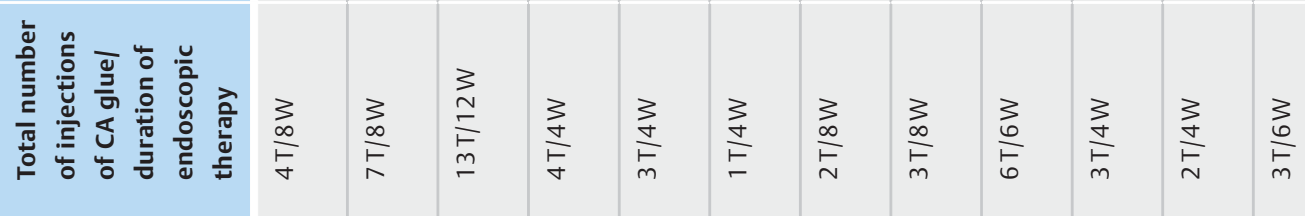
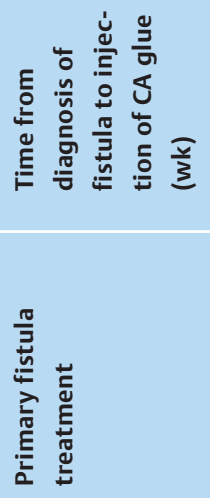

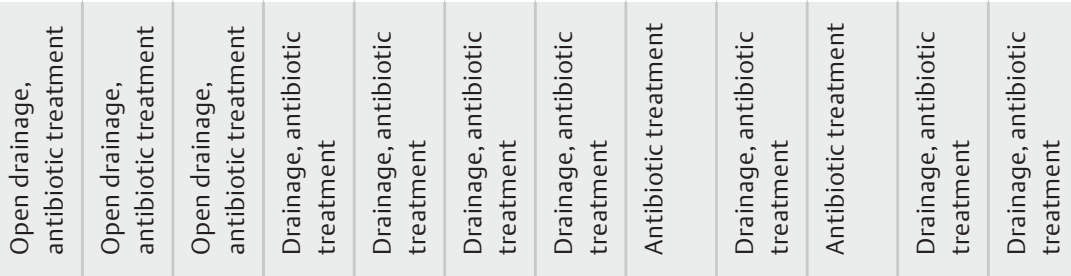
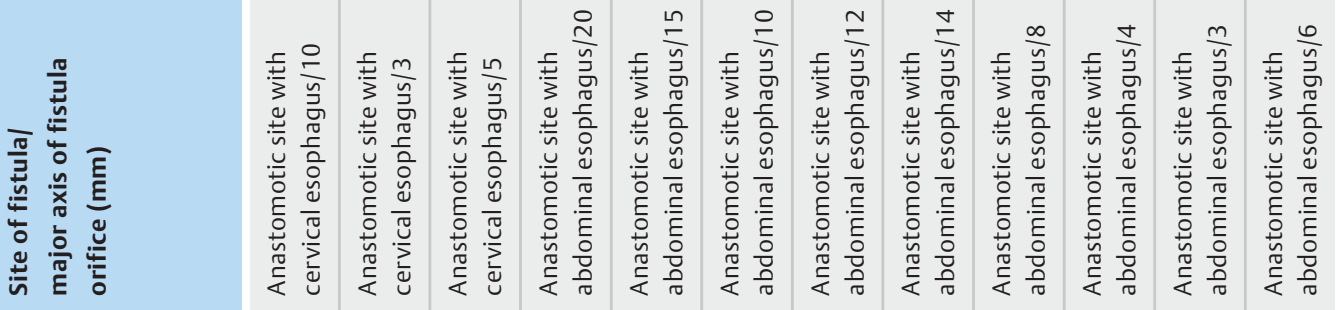

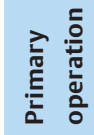
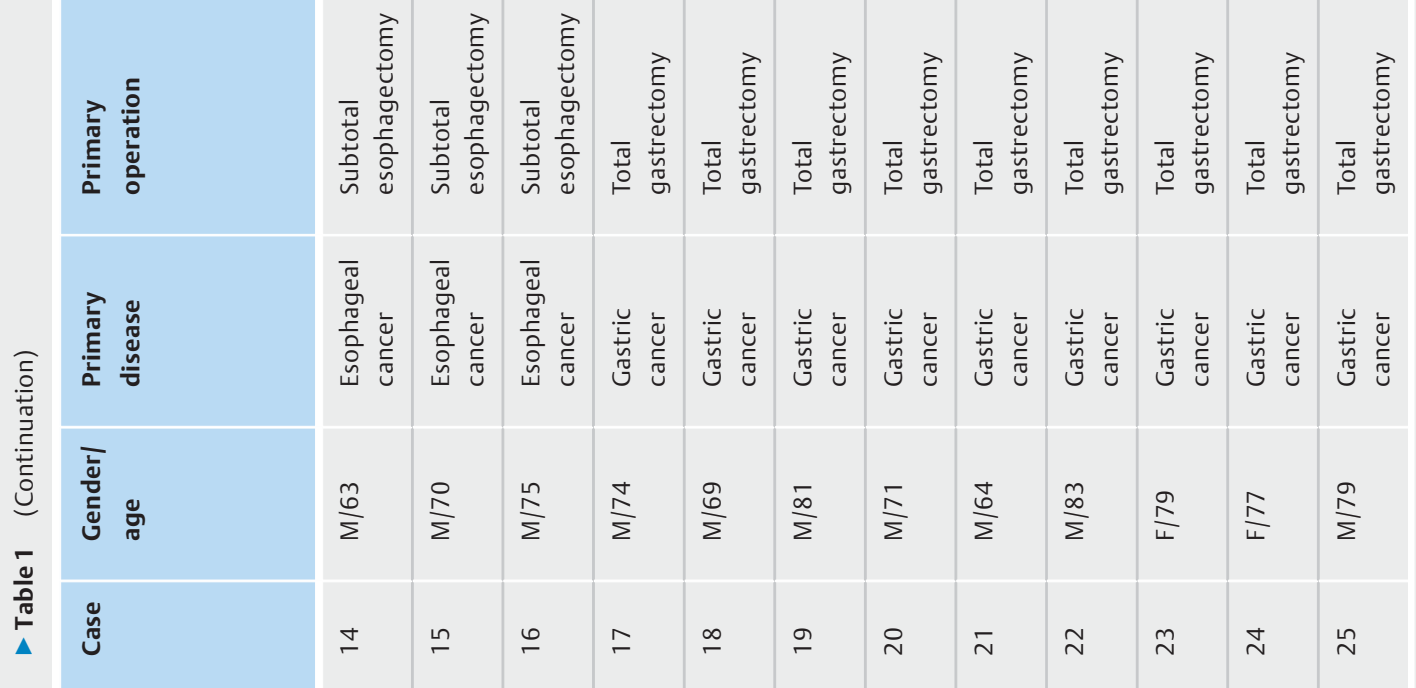

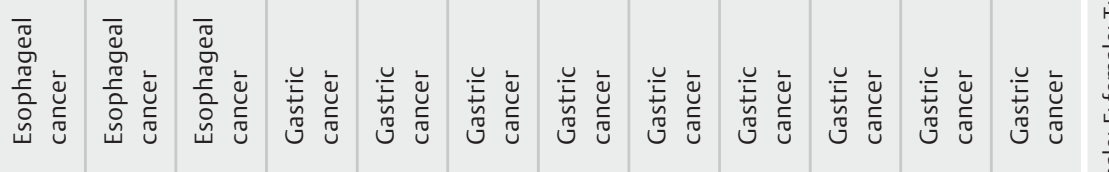

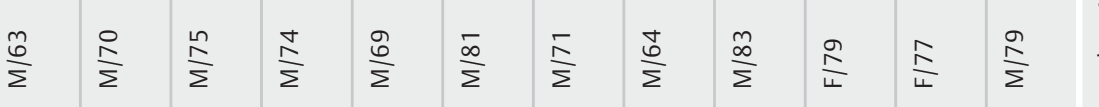

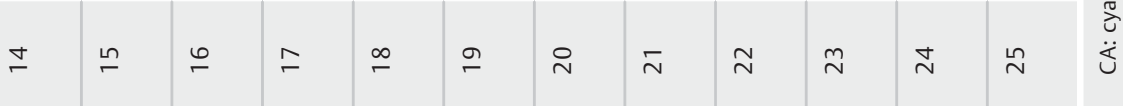




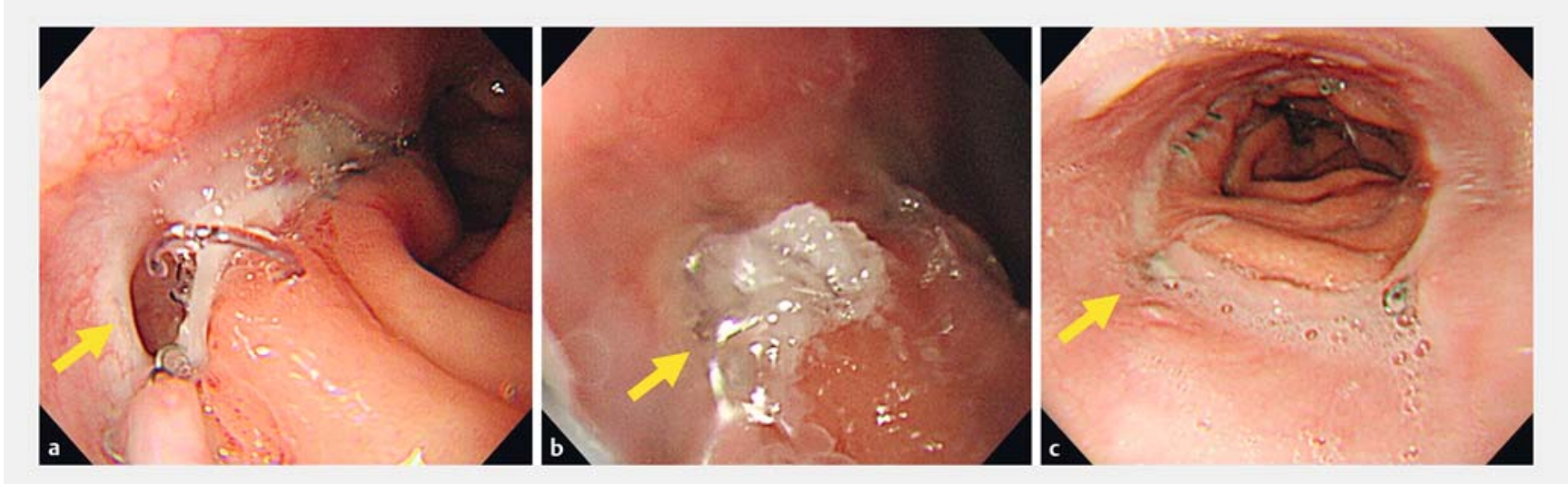

- Fig. 2 Endoscopic findings. a Endoscopic appearance of fistula opening. b A view of the fistula opening after cyanoacrylate glue injection. c Fistula closure. Yellow arrows show the fistula site.

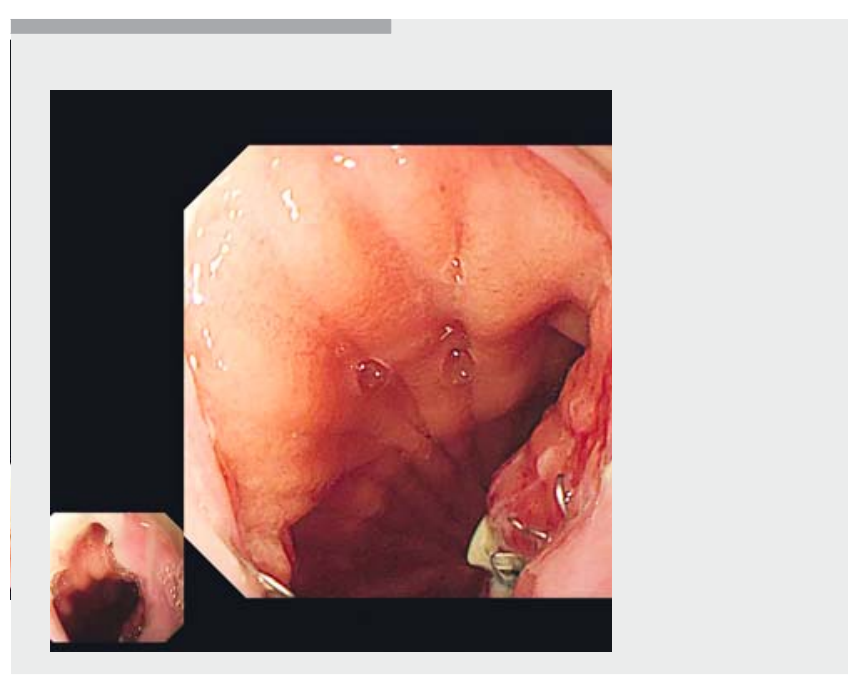

Video 1 Endoscopic treatment with injection of $\alpha$-CA monomer for esophageal fistulas.

age of cervical esophagus after the subtotal esophagectomy with esophagogastrostomy. The remaining 9 patients (36\%) had gastric cancer as primary disease. The cause of esophageal fistula was anastomotic leakage of abdominal esophagus after total gastrectomy with esophagojejunostomy. All patients received conservative medical treatment of over a week, such as fasting and antibiotic administration. In all 16 patients with anastomotic leakage of cervical esophagus after subtotal esophagectomy, we opened the cervical wound and noted the presence of fistula. In 7 of the 9 patients with an anastomotic leakage of abdominal esophagus after total gastrectomy, we performed percutaneous drainage of intra-abdominal abscess. The size of fistula orifice in the esophagus according to endoscopic findings was $8 \mathrm{~mm}$ in a median major axis (range: $3-20 \mathrm{~mm}$ ).

\section{Endoscopic treatments}

All patients were treated with endoscopic injection of $\alpha-C A$ monomer glue. Fig. 2 shows the pre- and posttreatment endoscopic findings of the fistula with the complete healing (case 19). The median duration from diagnosis of esophageal fistula to initiation of the endoscopic treatment was $2 \mathrm{wk}$ (range: $1-8 \mathrm{wk}$ ). The median number of injections of $\alpha$-CA monomer glue was 3 times (range: 1 - 14 times), and the median duration of endoscopic treatment was $6 \mathrm{wk}$ (range: 4-16 wk).

\section{Primary and secondary endpoints}

After treatment, complete closure of the fistula was verified in 22 patients by endoscope. Endoscopic injection of $\alpha$-CA monomer glue was ineffective in 3 patients. The overall success rate therefore was $88 \%(22 / 25)$. The 3 patients in which the treatment was ineffective were all esophageal cancer patients with cervical esophageal fistulas. They required reoperation of the fistulectomy plus muscle flap under general anesthesia. During the median follow-up period of 14 mo (range: 6 - 30 mo), 3 patients died of cancer, but the remaining patients completely recovered without recurrence of esophageal fistula.

In both early and late phases, there were no complications higher than Clavien-Dindo grade II in patients who received endoscopic treatments with injection of $\alpha$-CA monomer glue.

\section{Discussion}

This is the first prospective study of endoscopic treatment of an esophageal fistula using $\alpha$-CA monomer injection. In our study of 25 patients, complete closure of the esophageal fistula was possible in 22 patients after endoscopic injection of $\alpha-C A$ monomer; the overall success rate was $88 \%$. In addition, there were no complications associated with this endoscopic treatment and fistula recurrence. This phase II study met its primary endpoint of high fistula closure rate and its secondary endpoint of low complication rate. Therefore, we propose that endoscopic injection of $\alpha-C A$ monomer is a feasible and safe procedure for the treatment of nonhealing esophageal fistulas. 
Occurrence of esophageal fistula following esophagectomy is a severe complication because gastrointestinal contents drain into the thoracic or peritoneal cavities, leading to intrapleural abscess or peritonitis. The sealing of the fistulas orifice by endoscopic injection of superglue, which is exemplified by $\alpha-C A$ monomer, therefore, is an important step. In addition, $\alpha-C A$ monomer has the advantages of having both stronger adhesive properties and stronger antibacterial properties compared with other polymer glues, such as n-butyl 2-cyanoacrylate polymer and 2 -octyl cyanoacrylate polymer $[1,14]$. Therefore, $\alpha$-CA monomer glue is suitable for application in a wet and infected cavity. In this study, the treatment success rate of $88 \%$ is one of the best compared with previous retrospective studies $[1,10$, $11,15]$. In 3 case series with 22 [10], 10 [11], and 9 patients [15], respectively, the concomitant presence of fistula with this technique has proven to be associated with a lower success rate. Notably, n-butyl 2-cyanoacrylate polymer was used for glue embolization in the studies. We affirm that using $\alpha$-CA monomer would be more successful for this technique.

The injection of $\alpha$-CA monomer often causes endoscopic troubles because of the strong fast-acting adhesive characteristics associated with the superglue. In general, $\alpha$-CA monomer is an acrylic resin that rapidly polymerizes in the presence of a little water. Because the presence of moisture causes the glue to set, exposure to normal levels of humidity in the air causes polymerization to start within several seconds. Therefore, we mixed the $\alpha$-CA monomer and oily the contrast agent (lipiodol) at a ratio of 0.3 to $1.7 \mathrm{~mL}$ in a $2.5-\mathrm{mL}$ syringe. We developed this mixture ratio in terms of efficiency of the infusion [12]. Lipiodol has the added benefit of allowing radiological confirmation of the injection and identification of embolization to be performed. If the glue syringe containing the $\alpha$-CA monomer and lipiodol is made air-tight, we could endoscopically inject the solution safely in the image-guided therapy suite. On the other hand, it is generally accepted that the polymerization of $\alpha-C A$ monomer is delayed in the presence of a large quantity of water. Therefore, we used a $50 \%$ glucose solution to push the solution from the syringe into the endoscopic dispersion tube. In our experiments, $50 \%$ glucose solution was suitable for the pushing of the embolic solution [12]. We succeeded in performing the endoscopic injections of $\alpha$-CA monomer in all cases using the above-mentioned devices and methods.

In our study, the endoscopic treatment for esophageal fistulas closure failed in 3 patients (12\%). In these patients, the size of the fistula orifice in the esophagus was larger than $8 \mathrm{~mm}$ of the median $(8,10$, and $12 \mathrm{~mm}$, respectively). In addition, the fistula sites were all located in the flexion of the cervical esophagus. The hold of endoscopic position during this treatment was therefore difficult, and the infusion volume of $\alpha$-CA monomer glue during endoscopic treatment may be insufficient. The strong pressure exerted on the flexion of the cervical esophagus during swallowing may have caused the nonhealing fistulas. Furthermore, the systemic inflammatory reactions in failed patients were high. We postulate that stable patients who have fistulas in the abdominal esophagus after total gastrectomy with esophagojejunostomy may be managed by this endoscopic treatment. While the majority of patients with cervical esophageal fistulas after subtotal esophagectomy with esophagogastrostomy are candidates for this endoscopic treatment, it has its limitations in certain patients - for example, patients with fistula larger than $8 \mathrm{~mm}$, patients with fistula that render it difficult to hold an endoscopic position, and unstable patients with evidence of sepsis. In these patients, other endoscopic treatments, such as endoscopic clips and stent insertion, are also inadequate adaptations $[1,16]$. In our series, all 3 patients in which this endoscopic treatment was refractory were cured by surgical reinterventions with fistulectomy plus muscle flap. However, a more aggressive surgery, such as esophagectomy with proximal and distal stoma, is advisable before this endoscopic treatment is planned in unstable patients with a septic condition.

This phase II study had several limitations. It was conducted in a single institution without randomized controlled study. Due to the small sample size, findings from this do not allow established clinical application. Indeed, many patients with esophageal fistulas can be treated conservatively, and their fistulas may close during follow-up even when endoscopic intervention was not performed. Therefore, in our 22 success cases who received endoscopic injection of $\alpha$-CA monomer, the patients who were available for conservative treatment and the patients who really needed endoscopic treatment to make the fistula closure might be mixed. In order to prove the clinical application of this endoscopic treatment with injection of $\alpha$-CA monomer, we are going to plan a large multicenter, randomized, double-blind, placebo-controlled phase III clinical trial in the future.

In conclusion, endoscopic treatment with injection of $\alpha-C A$ monomer facilitated healing of post-esophagectomy fistula in $88 \%$ of patients without complications. This suggests that the treatment is effective and safe for patients with esophageal fistulas.

\section{Competing interests}

None

References

[1] Kotzampassi K, Eleftheriadis E. Tissue sealants in endoscopic applications for anastomotic leakage during a 25-year period. Surgery 2015; 157: $79-86$

[2] Dan DT, Gannavarapu B, Lee JG et al. Removable esophageal stents have poor efficacy for the treatment of refractory benign esophageal strictures (RBES). Dis Esophagus 2014; 27: 511 - 517

[3] Sharma P, Kozarek R, Practice Parameters Committee of American College of Gastroenterology. Role of esophageal stents in benign and malignant diseases. Am J Gastroenterol 2010; 105: 258-273

[4] Cameron R, Binmoeller KF. Cyanoacrylate applications in the Gl tract. Gastrointest Endosc 2013; 77: 846 - 857

[5] Barthelemy C, Audigier JC, Fraisse H. A non-tumoral esophago-bronchial fistula managed by isobutyl-2-cyanoacrylate. Endoscopy 1983; 15: $357-358$ 
[6] Yoon JH, Lee HL, Lee OY et al. Endoscopic treatment of recurrent congenital tracheoesophageal fistula with Histoacryl glue via the esophagus. Gastrointest Endosc 2009; 69: 1394-1396

[7] Rotondano G, Viola M, Orsini L et al. Uncommon cause of early postoperative colonic fistula successfully treated with endoscopic acrylate glue injection. Gastrointest Endosc 2008; 67: 183-186

[8] Iwata T, Chung K, Toda M et al. Malignant esophagobronchial fistula with severe esophageal stenosis, successfully treated by transbronchial occlusion with cyanoacrylate glue. Endoscopy 2012; 44 (Suppl. 02): E317-E318

[9] Katada K, Sano H, Katoh Y et al. Ethyl 2-cyanoacrylate as an embolic agent for cranial arteriovenous malformations. An experimental study. Acta Radiol Suppl 1986; 369: 623-626

[10] Willetts IE, Dudley NE, Tam PK. Endoscopic treatment of recurrent tracheo-oesophageal fistulae: long-term results. Pediatr Surg Int 1998; $13: 256-258$
[11] Billi P, Alberani A, Baroncini D et al. Management of gastrointestinal fistulas with n-2-butyl-cyanoacrylate. Endoscopy 1998; 30: S69

[12] Ojima T, Nakamori M, Nakamura M et al. Successful treatment of esophageal fistulas with endoscopic injection of alpha-cyanoacrylate monomer. Endoscopy 2014; 46 (Suppl. 01): E62 -E63

[13] Dindo D, Demartines N, Clavien PA. Classification of surgical complications: a new proposal with evaluation in a cohort of 6336 patients and results of a survey. Ann Surg 2004; 240: 205-213

[14] Muto G, D’Urso L, Castelli E et al. Cyanoacrylic glue: a minimally invasive nonsurgical first line approach for the treatment of some urinary fistulas. J Urol 2005; 174: 2239-2243

[15] Groitl H, Horbach T. Endoscopic treatment of anastomosis insufficiency and perforation in the esophagus with fibrin glue. Langenbecks Arch Chir Suppl Kongressbd 1996; 113: 753 - 754

[16] Christophorou D, Valats JC, Funakoshi N et al. Endoscopic treatment of fistula after sleeve gastrectomy: results of a multicenter retrospective study. Endoscopy 2015; 47: $988-996$ 